\title{
AVALIAÇĀO SISTEMATIZADA DO PROCESSO ENSINO-APRENDIZAGEM EM ENFERMAGEM MÉDICO-CIRÚRGICA
}

\author{
Miako Kimura* \\ Angela Maria Geraldo Pierin* \\ Cilene Aparecida Costardi Ide* \\ Eliane Correa Chaves* \\ Katia Grillo Padilha*
} Vera Lúcia Conceição de Gouveia Santos**

KIMURA, M.; PIERIN, A.M.G.; IDE, C.A.C.; CHAVES, E.C.; PADILHA, K.G.; SANTOS, V.L.C. de G. Avaliação sistematizada do processo ensino-aprendizagem em enfermagem médico-cirúrgica. Rev. Esc. Enf. USP, São Paulo, 20(1) :27-38, 1986.

O trabalho versa sobre as expectativas assim como a avaliafão dos discentes quanto ao processo ensino-aprendizagem desenvolvido pelos docentes da disciplina Enfermagem Médico-Cirúrgica do Departamento de Enfermagem Médico-Cirúrgica da Escola de Enfermagem da Universidade de São Paulo. São apresentadas, também, as sugestões referidas pelo grupo de alunos para o aprimoramento do ensino dessa disciplina.

\section{INTRODUÇÃO}

Os docentes da disciplina Enfermagem Médico-Cirúrgica (EMC), do Departamento do mesmo nome da Escola de Enfermagem da Universidade de São Paulo (EEUSP), têm como preocupação constante a avaliação da eficácia e coerência do processo ensino-aprendizagem. Desde 1981 sentiu-se a necessidade de avaliação mais objetiva, sobretudo por parte dos discentes, que fornecesse subsídios para o aprimoramento da disciplina. Assim sendo, ao término de cada curso, solicita-se a avaliação dos alunos, utilizando-se impresso específico.

Os resultados obtidos têm sido fonte importante para reestruturação do curso - metodologia de ensino, conteúdo programático e prática de campo - visando adequação do processo de ensino da disciplina. Acredita-se que esse processo venha subsidiar uma reformulação curricular mais ampla.

* Enfermeira. Mestre em Enfermagem. Professor Assistente do Departamento de Enfermagem Médico-Cirúrgica da Escola de Enfermagem da USP - disciplina Enfermagem Médico-Cirárgica.

* Enfermeira. Auxiliar de Ensino do Departamento de Enfermagem Médico-Cirúrgica da Escola de Enfermagem da USP - disciplina Entermagem Médico-Cirúrxica. 
KAMTYAMA (1982) enfatizou que «a reformulação do currículo deve ter como base a análise do papel da enfermeira na sociedade, de suas funções e do perfil do profissional esperado pela comunidade, fatores dinâmicos que se encontram em constante evolução».

CAMPOS apud KAMIYAMA (1982) afirmou que «o ensino profissional deve ser o precursor de novas tendèncias e aspirações científico-culturais e o professor, líder do processo educacional».

Ao analisar-se retrospectivamente o desenvolvimento do ensino da disciplina, verifica-se que em 1981 a abordagem teórico-prática era fundamentada em especialidades, tais como assistência de enfermagem em cardiologia, neurologia, urologia-renal, pneumologia e gastroenterologia. MIYADAHIRA et alii (1982), avaliando a consecução dos obbjetivos propostos, por discentes e docentes, concluíram que estes foram parcialmente atingidos. Nesta ocasião, as sugestões feitas ressaltavam a necessidade de mudança na estratégia de ensino até então utilizada.

Estes dados fundamentaram a reformulação ampla da estrutura do ensino da disciplina. Em 1982 a abordagem teórica, antes voltada para patologias de diferentes sistemas, passou a ser feita com base nos sinais e sintomas que caracterizam grande parte dos problemas de enfermagem. A utilização de um processo de ensino visando proporcionar oportunidades de experiências em niveis crescentes de complexidade, foi também incluida neste programa, além de duas novas sub-unidades: enfermagem em Unidade de Terapia Intensiva (UTI) e Reabilitação.

Ao final desse periodo, CHAVES et alii (1983) realizaram nova avaliação do ensino, observando-se que, para a maioria dos discentes e docentes, os objetivos haviam sido totalmente atingidos; a sugestão principal recaiu predominantemente na ampliação da carga horária da disciplina, não mais se evidenciando a necessidade de mudanças na estratégia de ensino.

Decorridos 3 anos da implementação das modificações citadas anteriormente, sentiu-se mais uma vez, a necessidade de se continuar o processo avaliativo em busca de novos dados para o aprimoramento do curso.

Para tanto o presente estudo tem, como objetivo identificar:

1 - as expectativas dos alunos no início do curso;

2 - os aspectos teórico-práticos do programa, citados pelos alunos, que necessitam de reforço;

3 - quais objetivos propostos pelos docentes da disciplina, foram atingidos;

4 - as sugestões dos discentes para o aprimoramento do ensino.

\section{METODOLOGIA}

O presente trabalho foi elaborado a partir da análise de duas fases distintas: a primeira, correspondente ao levantamento de expectativas e a segunda, à avaliação da disciplina pelos alunos. 


\section{População}

A população de estudo foi composta por: - 57 alunos $(75 \%$ da classe), presentes no primeiro dia letivo da disciplina EMC, em 1984, na $1^{\text {: }}$ fase; -54 alunos $(71 \%)$, na $2^{\text {a }}$ fase, presentes no último dia letivo da disciplina.

\section{Coleta de Dados}

Para a coleta de dados empregaram-se dois questionários: o primeiro, para o levantamento das expectativas e o segundo, para avaliação e sugestões.

O questionário utilizado na $1^{\text {a }}$ fase foi composto de duas questões abertas, que visavam obter as seguintes informações:

- as expectativas dos discentes quanto à disciplina EMC (questão 1) ;

- assuntos abordados em disciplinas anteriores que os alunos desejavam ver reforçados, quanto a procedimentos, fundamentação teórica e outros (questão 2).

O questionário utilizado na $2^{\mathrm{a}}$ fase abrangeu duas questões, objetivando coletar a avaliação do curso quanto a:

- consecução dos objetivos da disciplina EMC nas Unidades de Internação, nas Unidades de Terapia Intensiva e na de Reabilitação, a ser classificada em objetivos «totalmente atingidos, parcialmente atingidos ou não atingidos» (questão 1);

- justificativas apresentadas pelos discentes para a classificação dos objetivos atingidos, nas 3 Unidades (questão 1-a); (questão 2).

- sugestões relacionadas pelos alunos para o ensino da disciplina

\section{Tratamento dos Dados}

Os dados colhidos na primeira e segunda fases foram tabulados e distribuídos em tabelas, com freqüência absoluta e relativa, seguidas de análise e comentários.

\section{RESULTADOS E COMENTARIOS}

\section{Primeira fase - Expectativas}

O total de alunos que responderam ao questionário foi de 57, gerando ao todo 127 expectativas. Assim, cada aluno apresentou, em média, 2,3 expectativas quanto à disciplina.

Os dados da tabela 1 mostram que os alunos, antes de cursarem a disciplina EMC, esperam uma ampliação de conhecimentos teórico-práticos $(50,4 \%)$, comportamento este natural no início de cada nova disciplina no decorrer do Curso. 
TABELA 1

EXPECTATIVAS DOS DISCENTES QUANTO A DISCIPLINA

DE EMC. EEUSP, 1984.

\begin{tabular}{|c|c|c|}
\hline Expectativas & ne & $\%$ \\
\hline Aquisição de conhecimento teórico-prático & 64 & 50,4 \\
\hline $\begin{array}{l}\text { Reforço dos conhecimentos teórica-práticos } \\
\text { de Fundamentos de Enfermagem }\end{array}$ & 21 & 16,5 \\
\hline Segurança e maturidade na assistência global aos pacientes & 14 & 11,0 \\
\hline Liberdade no relacionamento docente/aluno & 13 & 10,3 \\
\hline Utilização de metodologia adequada & 8 & 6,3 \\
\hline Ampliação na visão da Enfermagem como profissão & 7 & 5,5 \\
\hline Total & 127 & 100,0 \\
\hline
\end{tabular}

Ao mesmo tempo, foi significativo o número de respostas sobre a necessidade de reforçar aspectos do ensino teórico-prático de disciplinas anteriormente cursadas $(16,5 \%)$.

Os discentes esperam, também, que a disciplina EMC os prepare para desempenho mais seguro na prestação da assistência global aos pacientes $(11,0 \%)$, o que poderá ser alcançado, em parte, mediante o acréscimo dos conhecimentos teórico-práticos, enfaticamente esperado por eles.

Outras preocupações foram manifestadas quanto a relacionamento com docentes $(10,3 \%)$ e metodologia a ser empregada no ensino $(6,3 \%)$. Apesar de numericamente pouco significativo $(5,5 \%)$, foi interessante encontrar, já nesta fase do curso, expectativas relativas à Enfermagem como profissão.

Confrontando-se as tabelas 2 e 3 , verifica-se que as expectativas dos discentes referem-se predominantemente ao reforço de procedimentos (122 respostas) sobre a fundamentação teórica (33 respostas), na proporção aproximada de 4 para 1 . Tanto no aspecto prático como no teórico, observa-se que a expectativa mais indicada foi referente às medicações: preparo e administração parenteral $(41,8 \%)$ e conhecimento, principalmente no que se refere ao cálculo $(48,5 \%)$.

Além deste item, procedimentos como sondagens vesical e nasogástrica $(22,1 \%)$ e enteroclisma $(21,3 \%)$ foram significativamente mencionados.

Quanto aos aspectos da fundamentação teórica, somente $15,1 \%$ das respostas foram sobre maior conhecimento de doenças, o que tem sido observado em índices bem mais elevados nos anos precedentes. 
TABELA 2

PROCEDIMENTOS QUE OS DISCENTES GOSTARIAM QUE FOSSEM REFORÇADOS, NA DISCIPLINA EMC. EWUSP, 1984.

\begin{tabular}{|c|c|c|}
\hline Procedimentos & $\mathbf{n}^{\circ}$ & $\%$ \\
\hline Preparo e administração de medicação & 51 & 41,8 \\
\hline Sondagens & 27 & 22,1 \\
\hline Enteroclisma & 26 & 21,3 \\
\hline Curativos & 8 & 6,5 \\
\hline Ataduras & 7 & 7,7 \\
\hline Outros * & 3 & 2,4 \\
\hline Total & 122 & 100,0 \\
\hline
\end{tabular}

* Outros: inclui drenagem de tórax, coleta de sangue e manipulação de aparelhos.

TABELA 3

ASPECTOS DA FUNDAMENTAÇAO TEORICA QUE OS DISCENTES GOSTARIAM QUE FOSSEM REFORÇADOS NA DISCIPLINA EMM. FEUSP, 1984.

\begin{tabular}{|c|c|c|}
\hline Fundamentação Térica & $\mathbf{n}^{9}$ & $\%$ \\
\hline Conhecimento sobre medicamentos* & 16 & 48,5 \\
\hline Conhecimento sobre doenças & 5 & 15,1 \\
\hline $\begin{array}{l}\text { Processo de Enfermagem } \\
\text { (ensino da sistematização - aplicabilidade ou não) }\end{array}$ & $\mathbf{5}$ & 15,1 \\
\hline Relacionamento enfermeiro/paciente & 2 & 6,1 \\
\hline Papel do Enfermeiro & 2 & 6,1 \\
\hline Outros ** & 3 & 9,1 \\
\hline Total & 33 & 100,0 \\
\hline
\end{tabular}

* Inclui cálculo de medicação: 14.

** Outros: inclui ética, problemas respiratórios e outros campos de atuaçăo da enfermagem.

Essa mesma porcentagem $(15,1 \%)$ foi obtida quanto ao reforço do ensino e da implementação do Processo de Enfermagem, como sistemática de assistência.

Na tabela 3 , assim como na tabela 1 , pode-se constatar novamente a referência ao papel do enfermeiro $(6,1 \%)$. 
Os resultados apresentados na tabela 4 indicam que a maioria dos discentes apontou os objetivos propostos pelos docentes da disciplina como parcialmente atingidos, em relação às Unidades de Terapia Intensiva e Reabilitação $(57,4 \%$ e $63,0 \%$, respectivamente). Já, com relação aos estágios nas Unidades de Internação, que constituem a maior ênfase

TABELA 4

AVALIAÇAO DOS DISCENTES QUANTO A CONSECUÇAO DOS OBJETIVOS PROPOSTOS PARA A DISCIPLINA EMC NA UNIDADE DE INTERNAÇAO, UTI E REABILITAÇAO. EEUSP, 1984.

\begin{tabular}{|c|c|c|c|c|c|c|c|c|}
\hline \multirow{3}{*}{ Locais } & \multicolumn{8}{|c|}{ Alcance dos } \\
\hline & \multicolumn{2}{|c|}{$\begin{array}{l}\text { Totalmente } \\
\text { atingidos }\end{array}$} & \multicolumn{2}{|c|}{$\begin{array}{c}\text { Parcialmente } \\
\text { atingidos }\end{array}$} & \multicolumn{2}{|c|}{ Não atingidos } & \multicolumn{2}{|c|}{ Total } \\
\hline & $n^{\circ}$ & $\%$ & $\mathrm{n}^{9}$ & $\%$ & $\mathrm{n}^{9}$ & $\%$ & $\mathbf{n}^{\mathbf{0}}$ & $\%$ \\
\hline $\begin{array}{l}\text { Em Unidade } \\
\text { de Internação }\end{array}$ & 36 & 66,7 & 18 & 33,3 & - & 0,0 & 54 & 100,0 \\
\hline Em UTI & 17 & 31,5 & 31 & 57,4 & 6 & 11,1 & 54 & 100,0 \\
\hline Em Reabilitação & 9 & 16,7 & 34 & 63,0 & 11 & 20,3 & 54 & 100,0 \\
\hline
\end{tabular}

da disciplina, os objetivos foram considerados totalmente atingidos por $66,7 \%$ dos alunos. Ressalta-se, ainda, que, nesta Sub-Unidade, não houve qualquer resposta quanto à não consecução dos seus objetivos. Em contraposição, um índice elevado de alunos $(20,3 \%)$ considerou que os objetivos da Unidade de Reabilitação não foram alcançados.

Os dados expressos na tabela 5 revelam que as maiores freqüências de justificativas para a consecução dos objetivos foram atribuídas às condições de desenvolvimento da prática em campo $(45,7 \%$ e $23,9 \%)$. Esses resultados, quando comparados aos da tabela anterior, parecem estar relacionados ao nivel de satisfação obtido pela maioria dos alunos por terem os objetivos sido atingidos totalmente em Unidades de Internação $(66,7 \%)$.

Alguns fatores detectados em menores índices, como falhas individuais $(15,2 \%)$, problemas referentes aos campos $(6,5 \%)$ e outros $(8,7 \%)$, justificariam a satisfação parcialmente manifestada por $33,3 \%$ dos alunos (tabela 4).

Através dos dados da tabela 6 , verifica-se que somente $23,9 \%$ das justificativas relaciona-se à consecução do objetivo específico desta Sub-Unidade, ou seja, a possibilidade de identificar problemas característicos do paciente crítico e da própria UTI.

A quase totalidade das demais respostas refere-se a periodo e época inadequados do estágio em UTT $(36,6$ e $18,3 \%$, respectivamente), o que o torna cansativo e estressante $(7,1 \%)$, não permitindo o alcance pleno de seus objetivos, como mencionado por $57,4 \%$ dos alunos (tabela 4). 
JUSTIFICATIVAS APRESENTADAS PELOS DISCENTES SOBRE O PORQUE DOS OBJETTVOS PROPOSTOS TEREM SIDO ATINGIDOS OU NAO NA UNIDADE DE INTERNAÇAO. EEUSP, 1984.

Justificativas

Estágio rico, diverso, completo, dinâmico, suficiente, com possibilidade de desenvolvimento de pesquisas

Possibilidade de aprendizado crescente (Identificação, Correlação, Planejamento e Assistência de Enfermagem)

Falha individual no aprofundamento teórico-prático

Problemas sobre os campos de prática:

(inadequação do campo, periodo insuficiente de estágio)

Outros * $\mathbf{n}^{\varphi}$

$\%$

21

45,7

11

23,9

7

15,2

3

\section{Total}

* Outros: problemas psico-sócio-espirituais pouco enfocados (1); heterogeneidade dos docentes (1); boa integração discente/docente (1); pouca correlação térico/ prática (1).

TABELA 6

JUSTIFTCATIVAS APRESENTADAS PELOS DISCENTES, SOBRE O PORQUE DOS OBJETTVOS PROPOSTOS TEREM SIDO ATINGIDOS OU NAO

NA UNIDADE DE TERAPIA INTENSIVA. EEUSP, 1984.

Justificativas

Periodo curto de estágio

Possibilidade de identificação de problemas específicos do paciente e da Unidade (incluindo atuação da enfermeira)

Epoca inadequada (cansaço)

Dificuldade individual em identificar e correlacionar problemas (pouca vivência, insegurança)

Estágio cansativo e estressante

Falta de atuação no atendimento em parada cardio-respiratoria

n* $\quad \%$


Ressalta-se, ainda que $12,7 \%$ das justificativas referem-se a dificuldades individuais (tabela 6).

Conforme se constata pela tabela 7 , o ensino em Reabilitação foi considerado enriquecedor e proveitoso $(20,6 \%)$; entretanto, fatores vários como problemas relativos ao campo $(19,1 \%)$, a falta de prestação de assistência pelo aluno $(14,7 \%)$, período curto de estágio $(8,8 \%)$, e outros, interferiram no aproveitamento dos alunos.

Nota-se, também, que, apesar da dificuldade em identificar o papel do enfermeiro na área de Reabilitação $(14,7 \%)$, alguns dos alunos $(5,9 \%)$ percebem a importância de sua atuação nessa área.

TABELA 7

JUSTIFICATIVAS APRESENTADAS PELOS DISCENTES, SOBRE O PORQUE DOS OBJETIVOS PROPOSTOS TEREM SIDO ATINGIDOS OU NAO NA UNIDADE DE REABIITACCAO. EEUSP, 1984.

\begin{tabular}{lcc}
\hline \multicolumn{1}{c}{ Justificativas } & $\mathrm{n}$ ^ & $\%$ \\
\hline $\begin{array}{l}\text { Estágio enriquecedor e proveitoso (reflexão dos problemas } \\
\text { sociais dos pacientes; experiência de vida) }\end{array}$ & 14 & 20,6 \\
$\begin{array}{l}\text { Problemas relacionados ao campo (contato, seleção } \\
\text { e distribuição) }\end{array}$ & 13 & 19,1 \\
Falta de prestação de assistência pelo aluno & 10 & 14,7 \\
Dificuldade em identificar o papel da enfermeira & 10 & 14,7 \\
Período curto de estágio & 6 & 8,8 \\
Fundamentação teórico-prática insuficiente & 4 & 5,9 \\
Percepção da importância da enfermeira & 4 & 5,9 \\
Pouco aproveitamento & 4 & 5,9 \\
Ausência do docente no campo & 3 & 4,4 \\
\hline \multicolumn{1}{c}{ Total } & 68 & 100,0 \\
\hline
\end{tabular}

A tabela 8 relaciona as principais sugestões apresentadas pelos discentes quanto à disciplina EiMC. Verifica-se que $78,2 \%$, de um total de 78 respostas, referem-se ao bloco teórico/prático, principalmente das Unidades UTT (mudança de época e período de estágio, 15,3\% e 12,8\%, respectivamente) e Reabilitação (aumento do período e aprofundamento do estágio, 12,8\%).

As sugestões concernentes à Avaliação (15,3\%) e ao Relaciona mento discente/docente $(6,5 \%)$ foram mencionadas em baixos índices percentuais. Os pontos citados nestes sub-itens distribuíram-se de maneira equitativa. 
TABELA 8

SUGESTÕES APRESENTADAS PELOS DISCENTES SOBRE O ENSINO

DA DISCIPLINA EMMC. EEUSP, 1984.

\section{Bloco teórico-pratico}

- Mudança de época do estágio em UTI

- Aumento do periodo de estágio em UTI

- Aumento do periodo e aprofundamento do estágio de Reabilitação

- Melhor distribuição do bloco teorico-prático

- Melhor seleção de campos de estágio

- Mais aulas práticas no Laboratório de Enfermagem

- Outras*

\section{Avaliagão}

- Avaliação dos docentes pelo aluno

- Avaliação por notas (manter)

- Inversão da ordem das provas

- Exigência maior de conhecimentos em fisiopatologia

- Falta nas atividades de ensino, sem prejuizo da avaliação

Relacionamento discente/docente

- Aberto e compreensivo

- Homogeneidade entre docentes

- Riqueza de experiências profissionais dos docentes

* Outras: abertura de campo para Enfermagem do Trabalho (1); reunióes após estágio (1); aulas teóricas especificas (3); orientação para a pesquisa (1); aula sobre problemática do paciente na UTI com abordagem dos aspectos psico-sócio-espirituais (1). 


\section{CONSIDERAgões FINAIS}

A disciplina EMC, desenvolvida no 49 semestre do Curso de Graduação em Enfermagem inicia o seu conteúdo teórico-prático a partir do embasamento das disciplinas do tronco pré-profissional e da primeira disciplina do tronco profissional comum, (Fundamentos de Enfermagem FE). Desta forma, após um ano de ensino essencialmente teórico, a prática em Enfermagem é finalmente iniciada e caberá ao aluno, no 2? ano, associar os dois conteúdos, apesar da defasagem de tempo que se estabeleceu entre estas duas etapas.

Competiria, portanto, às disciplinas FE e EMC a implementação do ensino básico da profissão no que se refere à abordagem do cliente hospitalizado, relacionamento com paciente, família e equipe multiprofissional, introdução do aluno à estrutura hospitalar e tudo o que ela representa, fundamentação teórico-prática das ações de enfermagem (incluindo procedimentos) que possibilitem a sistematização da assistência ao paciente adulto hospitalizado. Caberia, também, a esses docentes garantir aos estudantes oportunidade para questionarem e analisarem o ensino, a assistência e a realidade dos serviços de saúde utilizados como campos de estágio.

Teoricamente, no periodo de 8 meses letivos $\left(3^{\circ}\right.$ e $4^{\circ}$ semestres do curso), deveria ser desenvolvido pelos docentes e vivenciado pelos discentes, todo o conteúdo teórico-prático básico capaz de habilitar o aluno a assistir ao adulto doente, além de subsidiar o ensino de outros conhecimentos específicos, a serem ensinados nos anos subseqüentes, tais como: assistência de enfermagem à criança, à gestante, ao doente psiquiátrico, culminando na administração de unidades hospitalares.

Este quadro retrata a responsabilidade delegada aos docentes do $3^{\circ}$ e $4^{9}$ semestres do curso de Enfermagem, assim como a disparidade entre o tempo disponivel e a complexidade desse programa. Parece refletir, ainda, a inadequação curricular que propicia ao aluno o contato com a prevenção secundária antecedendo a primária, podendo gerar conflitos decorrentes dessa inserção na realidade hospitalar numa fase, ainda, de imaturidade profissional.

Essa situação configura, portanto, o compromisso e o risco de se introduzir o aluno, numa fase preliminar de formação, diretamente na assistência à pessoa hospitalizada, responsabilizando-se (docentes e discentes) pela atenção a pacientes com a integridade física comprometida, tanto pela afecção de base como pelos procedimentos invasivos utilizados no diagnóstico e na terapêutica, além dos problemas psico-sócio-espirituais decorrentes da doença e hospitalização.

A percepção dessa problemática, mesmo que parcial, parece justificar a preocupação referida pelos alunos quanto ao predomínio das expectativas quanto aquisição, desenvolvimento e fundamentação de habilidades básicas, sobrepujando aspectos intrínsecos do ensino e da profissão (relacionamento inter-pessoal, metodologia da assistência, visão da profissão como um todo). 
A valorização da experiência prática também se evidenciou quando se estabeleceu uma relação entre as características do campo de estágio e a possibilidade de aprendizado. Assim sendo, a utilização de «campos ricos, diversos e dinâmicos» (tabela 5), foram as características mais citadas para justificar a satisfação quanto aos estágios desenvolvidos em unidades de internação e que constituem a essência do conteúdo teórico-prático de EMC.

Outro aspecto a ser analisado refere-se à defasagem entre as expectativas dos estudantes e os objetivos dos docentes, quanto aos estágios em UTI e Reabilitação. Para os professores, a complementação do aprendizado a partir do conhecimento da assistência prestada tanto ao paciente em estado crítico (UTI), como àquele que necessita de reabilitação, se faz ao final do curso. A finalidade básica das duas sub-unidades é propiciar ao estudante a percepção de que a assistência secundária, desenvolvida nas unidades de internação, não se encerra em si mesma e que novos e complexos conhecimentos e habilidades são necessários; contudo, a amplitude dessa assistência faz com que o objetivo dos docentes limite-se, primordialmente, a mostrar essa realidade e não a habilitar o aluno a dominá-la. Talvez seja esse o principal fator para justificar a satisfação apenas parcial dos objetivos dos discentes, nessa fase em que esperavam estágio maior.

A validade das experiências desses campos, contudo, foi significativamente relatada, demonstrando que, apesar das limitaçōes de período, duração e atividades desenvolvidas, esses estágios foram valorizados por possibilitarem o enriquecimento do aprendizado.

Finalizando, pode-se perceber que os discentes do $4^{\circ}$ semestre do curso de Enfermagem esperam o aprimoramento téórico-prático na aquisição de habilidades básicas para a assistência de enfermagem e desejam que esse conteúdo seja desenvolvido de maneira gradativa e de forma não cansativa.

Outros aspectos, intrínsecos à profissão, tais como o questionamento quanto ao papel do enfermeiro e sua atuação dentro do sistema de saúde, a discussão quanto ao compromisso e valor da metodologia de assistência parecem fugir do âmbito de percepção e análise da maioria dos discentes, talvez porque suas preocupações diante da amplitude da situação vivenciada dificultem o envolvimento com problemas mais amplos.

\section{CONCLUSÕES}

Os resultados do estudo realizado com os discentes de EMC, em 1984, conduziram às seguintes conclusões:

- o maior número de expectativas referidas caracterizaram o interesse na possibilidade de EMC fornecer acréscimo $(50,4 \%)$ ou reforço $(16,5 \%)$ de conhecimento teórico-prático, assim como maior segurança e maturidade na assistência ao paciente $(11,0 \%)$; as demais expectativas citadas, o relacionamento interpessoal $(10,3 \%)$ e a utilização de 
metodologia adequada $(6,3 \%)$ poderiam favorecer a satisfação daquela primeira expectativa;

- no que se refere ao reforço de conhecimentos detectou-se que o procedimento mais requisitado foi o preparo e administração de medicação $(41,8 \%)$, coincidentemente com a maior solicitação de fundamentação teórica, também relacionada a medicações $(48,5 \%)$;

- para a maioria dos discentes os objetivos propostos pelos docentes da disciplina foram totalmente atingidos nos estágios em unidades de internação $(66,7 \%)$ e parcialmente atingidos tanto nas UTIs $(57,4 \%)$ como nas de Reabilitação $(63,0 \%)$;

- o maior número de sugestões de discentes para o aprimoramento de EMC referem-se à adequação do bloco teórico-prático $(78,2 \%)$. Do restante, $15,3 \%$ dizem respeito a alterações na forma de avaliação e $6,5 \%$ ao relacionamento entre estudantes e docentes.

KIMURA, M.; PIERIN, A.M.G.; IDE, C.A.C.; CHAVES, E.C.; PADILHA, K.G.; SANTOS, V.L.C. de G. Systematized evaluation of the teaching-learning process in medical-surgical nursing. Rev. Esc. Enf. USP, São Paulo, 20(1):27-38, 1986.

This is a study about the students about the teaching - apprenticeship process used for Medical-Surgical Nursing at the University of Säo Paulo School of Nursing. and their evaluation of the program. It also contains students' suggestion on the tcaching method employed.

\section{REFERENCIAS BIBLIOGRAFICAS}

CHAVES, E.C. et alii. Fnfermagem médico-cirargica: seguimento sistematizado do ensino. In: CONGRESSO BRASILEIRO DE ENFERMAGEM, 35॰, Săo Paulo, 1983. 20p.

RAMIYAMA, Y. Consideraçós sobre o perfil do graduado em enfermagem. Rov. Fac. Knf. USP, São Paulo, 15(2):211-221, ago. 1981.

MIYADAHIRA, A.M.K. et alli. Avaliação por docentes $\theta$ alunos, do processo de integração das disciplinas enfermagem médica e enfermagem cirúrgica. Rov. Esc. Int. USP, Sð̃o Paulo, 16(2) :193-209, ago. 1982. 